\title{
Gravity Effects on Fluid Front Dynamics during Mold Filling
}

\author{
K. A. Olivero And M. C. Altan ${ }^{1}$ \\ School of Aerospace and Mechanical Engineering \\ University of Oklahoma \\ 865 Asp. Avenue, Room 212 \\ Norman, OK 73019-0601
}

\begin{abstract}
The effect of gravity on free surface shape during the filling of a disk-shaped cavity is experimentally studied. A disk-shaped mold cavity is constructed to measure spreading of the fluid front due to gravity. Fluid front sensors are mounted on the top and bottom mold walls at three radial locations. The time the fluid front reaches these sensor locations is recorded. This data is used to calculate the radial distance between the top and bottom of the fluid front at each sensor location. The important parameters that govern the fluid front dynamics are identified as the Reynolds, Bond, and Capillary numbers. Spreading is found to be mainly dependent on the Bond number, such that a large change in spreading is observed due to a small change in Bond number. Spreading increases with decreasing Capillary number and remains nearly constant over the range of Reynolds numbers studied.
\end{abstract}

\section{INTRODUCTION}

$\mathbf{M}$ OLDING OPERATIONS INVOLVING polymers, e.g., Reaction Injection Molding (RIM), are commonly used to manufacture net-shape components quickly and inexpensively. Molding is usually characterized by the displacement of air in a cavity by a polymeric resin which is either cooled or cured to form the final solid part. During filling, the interface between the polymer and the air, referred to as the free surface, progresses through the cavity. It is commonly known that the dynamics of this free surface can affect heat transfer as well as curing rate in the mold, and thus the mechanical properties of the molded part.

A number of experimental and theoretical studies on free surface shapes are published in literature. The most common approach in determining free surface shapes in molding processes is to assume the Capillary number is high and that viscous forces dominate surface forces. For instance, in Behrens et al. [1] the fluid front shape is completely dictated by the flow kinematics and gravity effects are

\footnotetext{
${ }^{1}$ Author to whom correspondence should be addressed.
} 
not considered. Other studies dealing with free surface shape include research on the motion of contact lines [2,3]. These address the issue of the viscous stress singularity and so-called slip length used in analysis of moving contact lines, but do not address the free surface shape over the entirety of the free surface. Closely related are investigations in determination of the dynamic contact angles [4-6]. Again, the focus of such studies is on the flow dynamics near the contact point. Blake investigated gravity effects on free surface shape in mold filling $[7,8]$; however this study involves a vertically aligned cavity in which gravity does not cause an asymmetric fluid front. Several studies have been performed which include gravity effects on the spreading of a liquid drop on a solid surface which do not include gravity effects on the shape and motion of the free surface (e.g., Chen et al. [9] and Hocking [10]), but not in a mold cavity.

A trend in molding processes is towards the use of lower viscosity resins. These resins allow for higher fill rates and lower injection pressures, thus leading to higher production rates and lower equipment costs. One such resin, Shell Epon $815 \mathrm{c}$, has a viscosity of only $500-700 \mathrm{cp}$ and is suitable for injection molding and resin transfer molding parts at relatively low injection pressures. However, the use of low-viscosity polymers increases the complexity of flow dynamics. Molding with such polymers may lead to Reynolds numbers that are too large for Stokes

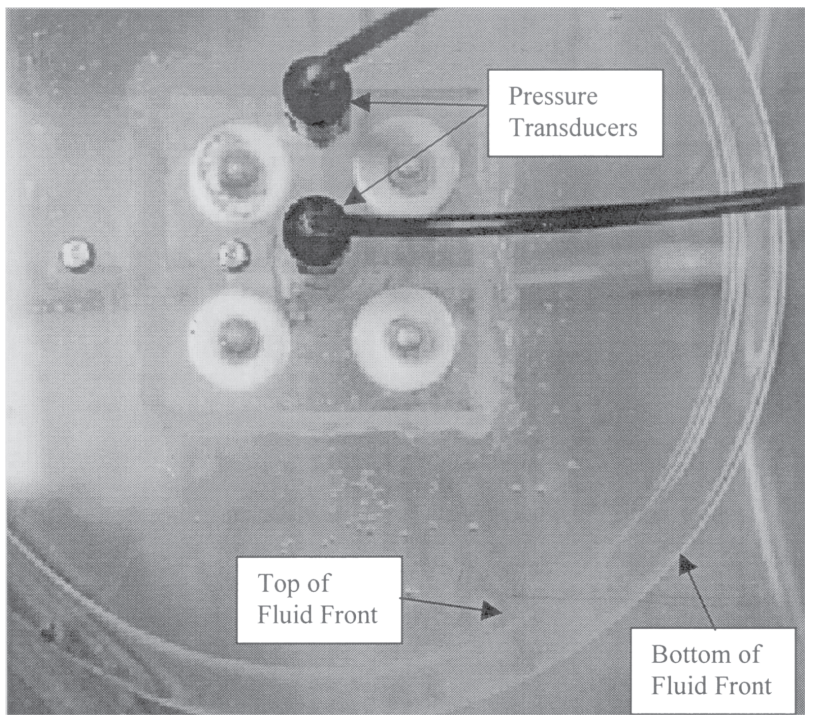

(a)

Figure 1. Spreading observed during filling of a mold cavity with corn syrup: (a) $\mu=412 \mathrm{cp}$, $\mathrm{Q}=2.13 \times 10^{-5} \mathrm{~m}^{3} / \mathrm{s}, 4.76 \mathrm{~mm}$ (0.1875 in.) gap-width, (b) $\mu=113 \mathrm{cp}, \mathrm{Q}=8.69 \times 10^{-5} \mathrm{~m}^{3} / \mathrm{s}$, $6.35 \mathrm{~mm}$ (0.25 in.) gap-width. 


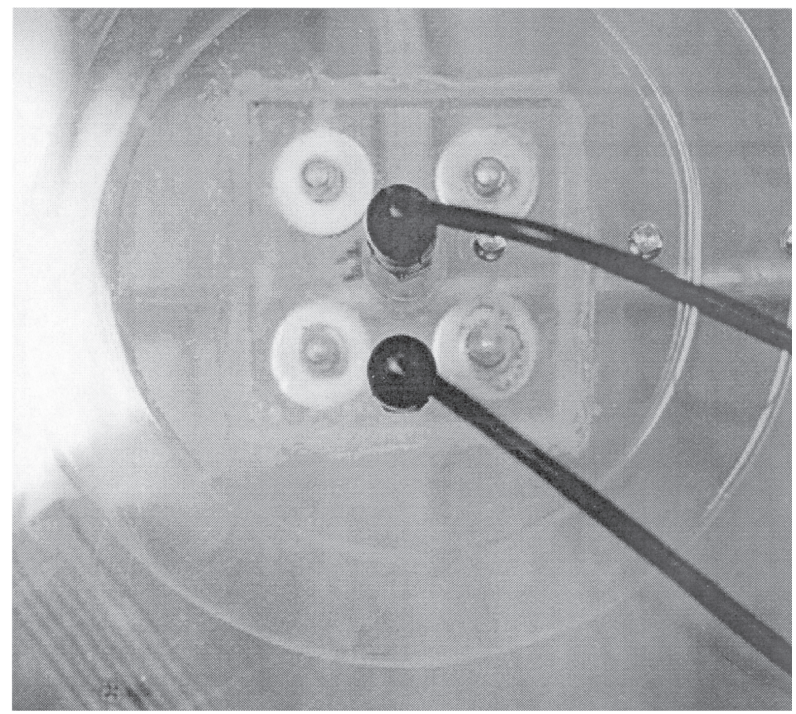

(b)

Figure 1 (continued). Spreading observed during filling of a mold cavity with corn syrup: (a) $\mu=412 c p, Q=2.13 \times 10^{-5} \mathrm{~m}^{3} / \mathrm{s}, 4.76 \mathrm{~mm}$ (0.1875 in.) gap-width, (b) $\mu=113 \mathrm{cp}, \mathrm{Q}=8.69 \times$ $10^{-5} \mathrm{~m}^{3} / \mathrm{s}, 6.35 \mathrm{~mm}(0.25 \mathrm{in}$.) gap-width.

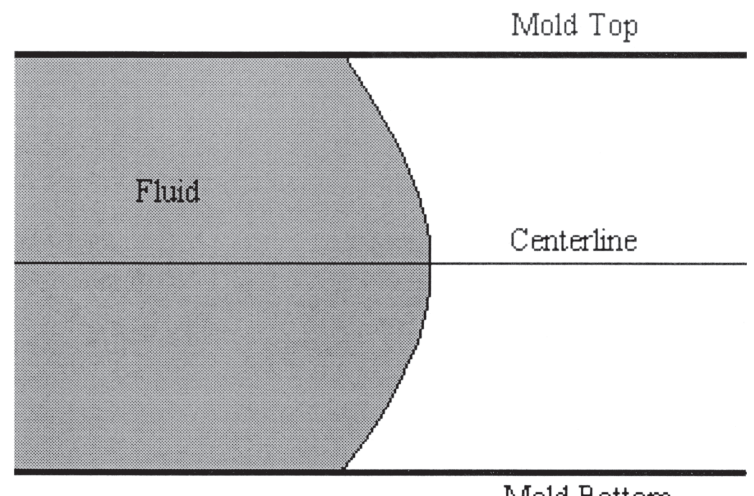

(a)

Figure 2. Representative fluid front shapes: (a) symmetric fluid front in the absence of gravity effects, (b) spreading of the bottom of fluid front due to gravity. 


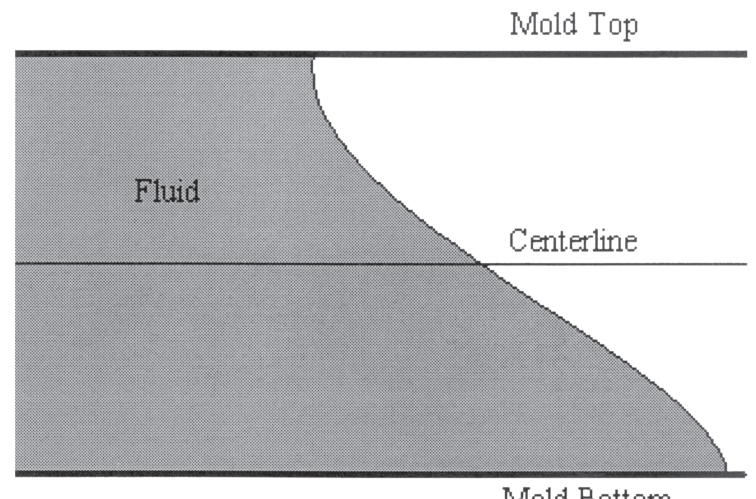

(b)

Figure 2 (continued). Representative fluid front shapes: (a) symmetric fluid front in the absence of gravity effects, (b) spreading of the bottom of fluid front due to gravity.

flow assumptions to be valid. For example, filling a disk-shaped cavtiy [76.2 $\mathrm{mm}$ (3 in.) radius, $3.175 \mathrm{~mm}$ (0.125 in.) thick] in eight seconds using Epon $815 \mathrm{c}$ resin yields a Reynolds number greater than 5. In addition, gravity effects on the free surface, which are usually neglected, can have pronounced effects on these flows, particularly on filling pattern, residence times, and weld line formation. The importance of mold orientation and the gravity effects in resin transfer molding of complex aircraft structures are discussed by Brosius and Wadsworth [11]. For planar mold cavities the flow is typically assumed to be symmetric about the midplane. However, if gravity effects become important, the bottom of the flow front (the outer circle in Figure 1) can advance well ahead of the top (the inner circle in Figure 1). Example fluid front shapes with and without sagging due to gravity are depicted in Figure 2.

The purpose of the current study is to experimentally study the effect of gravity on the free surface shape during the filling of a mold cavity. It is of particular interest to determine the important non-dimensional parameters governing the fluid front dynamics. Towards this end, mold filling experiments are performed to characterize the spreading of fluid front during the filling of a disk-shaped cavity. The effects of Reynolds, Bond, and Capillary numbers on spreading are studied.

\section{EXPERIMENTAL SETUP}

An experimental setup is constructed to observe and measure the features induced by gravity during the filling of a mold cavity. A disk shaped mold cavity is formed by placing aluminum spacer plates between two one-inch-thick Plexiglas sheets. A circular section with a $228.6 \mathrm{~mm}$ (9 in.) radius is cut out from the center 
of the spacer plates, thus forming a disk-shaped cavity. Various gap widths ranging from 1.59 to $25.4 \mathrm{~mm}$ (0.0625 to 1.0 in.) are attainable by combining spacer plates having thicknesses of $1.59,3.18,6.35,9.52$, and $12.7 \mathrm{~mm}(0.0625,0.125$, $0.25,0.375$, and 0.50 in.). Shim steel sheets having thicknesses of $0.10,0.20$, and $0.30 \mathrm{~mm}(0.004,0.008$, and $0.012 \mathrm{in}$.) are inserted between the spacer plates and Plexiglas to achieve more precise gap widths. Inlet gate diameters of 3.18, 6.35, $9.52 \mathrm{~mm}(0.125,0.250$, and 0.375 in.) can be selected by inserting one of three available inlet gates fabricated from Plexiglas. Pressure transducers and fluid front sensors for flow measurements are mounted along radial lines as illustrated in Figure 3. The advancing fluid front is sensitive to the surface quality of the mold walls, thus, the Plexiglas mold walls are sanded at the sensor locations by a series of 400, 600, 1000, and 4000 grit sandpaper, followed by polishing with a buffing wheel and compound. This greatly reduces perturbations to the fluid front shape as the front passes the sensor locations.

Glycerol diluted with water to obtain desired properties is used as the filling fluid. The fluid is injected at constant flow rate by a Masterflex IP73 microprocessor controlled peristaltic pump. Two pump heads can be configured in a parallel arrangement to yield flow rates for water ranging from $4 \times 10^{-6} \mathrm{~m}^{3} / \mathrm{s}$ to $2.7 \times 10^{-4}$ $\mathrm{m}^{3} / \mathrm{s}$ at a maximum pressure of $275 \mathrm{kPa}$ (40 psi). For higher viscosity fluids, the flow rates are somewhat reduced. After exiting the pump, the flow passes through a pulse dampener in order to minimize fluctuations in the flow which are common to peristaltic pumps. Each of these components is labeled on the photograph of the experimental setup shown in Figure 4.

Figure 5 is a close up diagram of one of the three fluid front sensors which are mounted in the mold cavity at $R=50.8,101.6$, and $152.4 \mathrm{~mm}(2,4$, and $6 \mathrm{in}$.). Each sensor consists of 4 terminals as shown in Figure 5: one power terminal and three

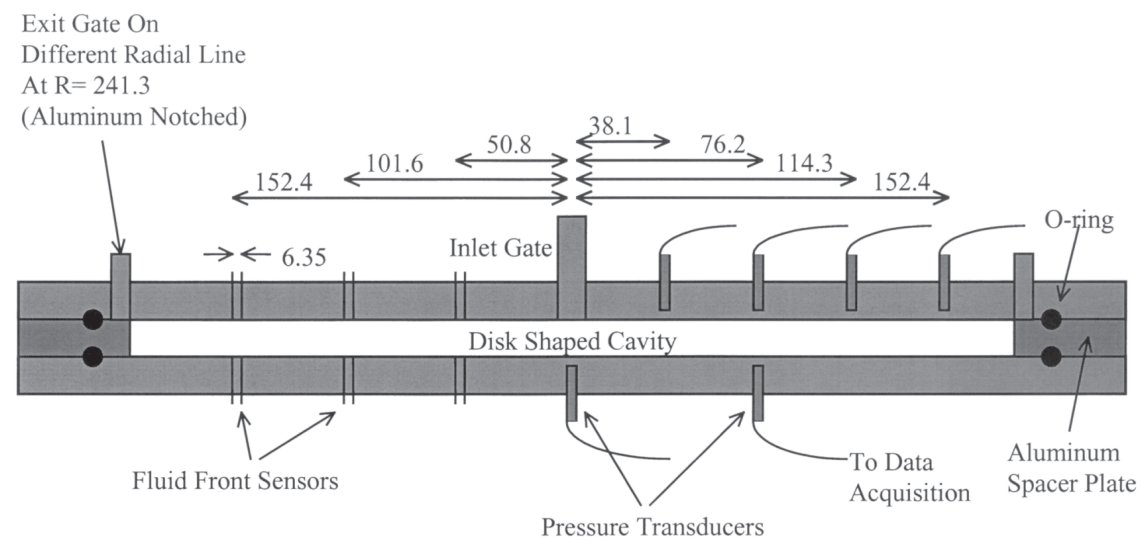

Figure 3. Experimental mold cavity diagram: cross section and dimensions. 


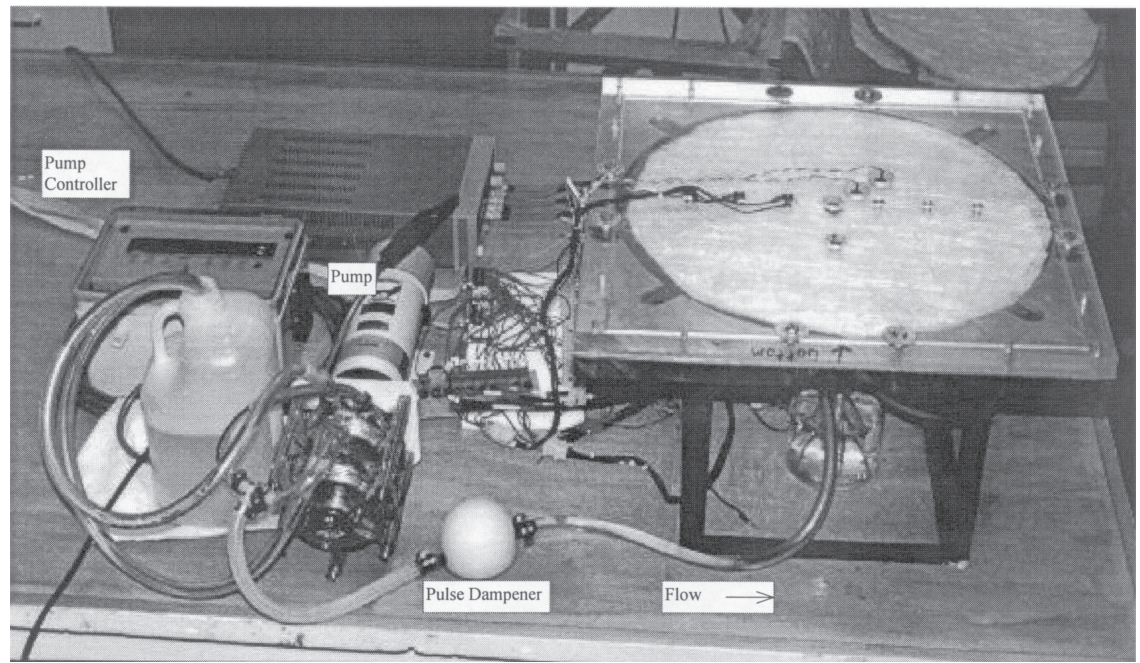

Figure 4. Setup for mold filling experiments. The mold is shown with three pairs of fluid front sensors and two pressure transducers placed on the top wall.

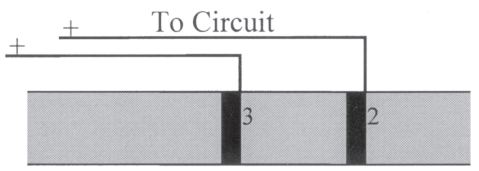

Top Probe

Mold Cavity

Bottom Probe

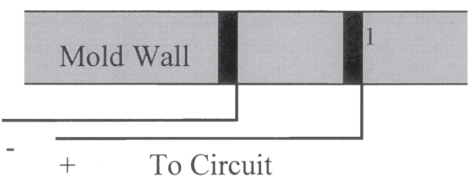

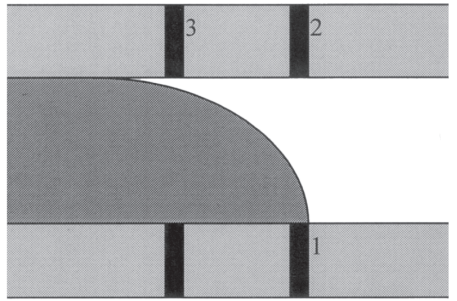

Figure 5. Schematic cross section of probe wiring depicting the advancement of fluid front through a probe location. 

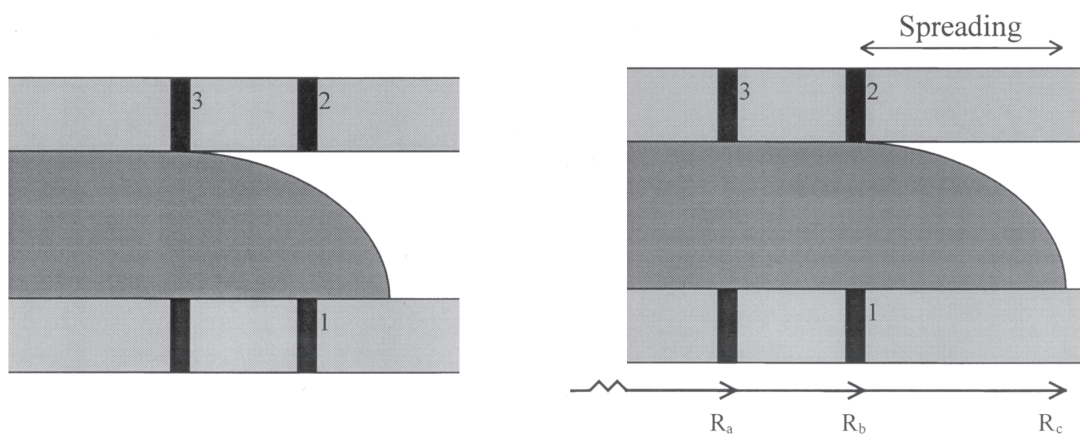

Figure 5 (continued). Schematic cross section of probe wiring depicting the advancement of fluid front through a probe location.

sensing terminals. A sensing circuit is connected to a data acquisition system to measure contact times based on the corresponding resistance drop when the fluid touches each sensing terminal. The circuit measures the change in resistance by measuring the voltage change across a reference resistor in series with the sensing terminal. An op amp in the circuit amplifies the voltage reading and conditions the signal by significantly lowering its output impedance (the data acquisition system has a maximum input impedance of $1000 \mathrm{ohms}$ ). A total of nine such circuits are attached to each of the sensing terminals. The three contact times are clearly identifiable in the raw voltage data obtained from each of the three fluid front probes.

\section{CALCULATIONS OF SPREADING FROM FLUID FRONT SENSOR DATA}

The spreading at each radial location is calculated from the times at which the fluid front contacts terminals 1,2 and 3 of a given fluid front probe. The first step in the spreading calculation is to calculate the velocity at the radius $R=R_{b}$ from two of the terminal contact times, $t_{2}$ and $t_{3}$. This is the time span over which the top of the fluid front travels between the top two terminals at $R=R_{a}$ and $R=R_{b}$. For this calculation, the free surface is assumed to travel at the average velocity of the flow for any given radius. The fluid is injected at a constant flow rate, leading to an average velocity inversely proportional to the radius,

$$
V(r)=\frac{d r}{d t}=\frac{c}{r}
$$

in which $c$ is a constant. Integrating this expression from $R_{a}$ to $R_{b}$ and from $t_{3}$ to $t_{2}$ respectively yields, 


$$
\frac{1}{2}\left(R_{b}^{2}-R_{a}^{2}\right)=c\left(t_{2}-t_{3}\right)
$$

The constant is determined from Equation (1) to be $c=V_{b} R_{b}$. This is substituted into Equation (2) and rearranged to yield an expression for the velocity at $b$,

$$
V_{b}=\frac{\left(R_{b}^{2}-R_{a}^{2}\right)}{2 R_{b}\left(t_{2}-t_{3}\right)}
$$

Equation (1) can also be integrated for the bottom of the fluid front, which travels from $R_{b}$ to $R_{c}$ over the time interval from $t_{1}$ to $t_{2}$. This integration is used to determine the final radius, $R_{c}$.

$$
R_{c}=\sqrt{R_{b}^{2}+2 V_{b} R_{b}\left(t_{2}-t_{1}\right)}
$$

The spreading is defined as the difference between the location of the top and bottom of the fluid front at time $t_{2}$

$$
S=R_{c}-R_{b}
$$

This value is calculated from the measured contact times for each probe.

\section{EXPERIMENTAL DESIGN}

The experimental setup is designed to simulate mold filling operations over a wide range of flow parameters. Dependence of spreading on these physical filling parameters, such as flow rate and gap width, can be easily ascertained by varying the individual parameter in question. However, it is useful to obtain the dependence of flow attributes on the non-dimensional parameters governing the flow dynamics. This is difficult to achieve by arbitrarily selected fill conditions, as changing a single parameter, such as the volume flow rate, can result in changes in all the non-dimensional parameters.

There are three non-dimensional parameters expected to affect spreading and flow characteristics during an isothermal, Newtonian filling: the Reynolds, Bond, and Capillary numbers. A fourth non-dimensional value, the contact angle, is a characteristic of the experimental apparatus (i.e., the contact angle depends on the fluid injected and the mold surface). Contact angle measurements for glycerol-water mixtures on Plexiglas are found to be between 0 and $38^{\circ}$ [12]. The commonly used resins in RTM process, such as Shell Epon and Dow Derakane, have similar contact angles ranging from 0 to $40^{\circ}$. The dependence of spreading on each of these non-dimensional parameters individually is desired. Thus, a method 
needs to be developed to determine the physical experimental parameters, i.e., the gap width, $L$, the volume flow rate, $Q$, and the volume fraction of glycerol in water, $f$, to achieve a prescribed set of non-dimensional parameters. To obtain this solution, the Reynolds, Bond, and Capillary numbers are defined as

$$
\begin{gathered}
\operatorname{Re}=\frac{\rho u L}{\mu} \\
B o=\frac{\rho g L^{2}}{\sigma} \\
C a=\frac{\mu u}{\sigma}
\end{gathered}
$$

where $g$ is the acceleration due to gravity, and $\rho, \sigma$, and $\mu$ are the fluid properties density, surface tension, and viscosity, respectively. These values are defined at the location of the first fluid front probe $(R=50.8 \mathrm{~mm})$, and thus the velocity, $u$, represents the average velocity at $R=50.8 \mathrm{~mm}$. The velocity, $u$, scales linearly with the volume flow rate, $Q$, and represents a characteristic velocity scale. These expressions are rearranged to isolate the unknown physcial quantities $u, L$, and $\mu$, for the desired set of Re, $B o$ and $C a$ :

$$
\begin{gathered}
\mu=\frac{\rho u L}{\operatorname{Re}} \\
u=\frac{\sigma C a}{\mu} \\
L=\left(\frac{\sigma B o}{\rho g}\right)^{1 / 2}
\end{gathered}
$$

Equations (10) and (11) are substituted into Equation (9) and arranged to yield

$$
0=\left(\frac{\rho B o}{g}\right)^{1 / 4}\left(\frac{C a}{\operatorname{Re}}\right)^{1 / 2}(\sigma)^{3 / 4}-\mu
$$

Gravity is a known constant and $\mathrm{Re}, \mathrm{Ca}$, and $\mathrm{Bo}$ are constant parameters, leaving three unknowns in Equation (12). It is observed that all unknown parameters in Equation (12) (i.e., $\mu, \rho$, and $\sigma$ ) are properties of the filling fluid. In this study, glycerol diluted with distilled water is used as the filling fluid, and the fluid proper- 
ties are obtained experimentally based on the volume fraction of glycerol in the mixture. These properties are also dependent on the temperature of the mixture. All measurements and experiments are performed at $25^{\circ} \mathrm{C}$. The density of pure glycerol, $\rho_{g}$, is measured to be $1256 \mathrm{~kg} / \mathrm{m}^{3}$ using a GARDCO specific gravity cup and an Ohaus TS4KD electronic balance. The density of distilled water at $25^{\circ} \mathrm{C}, \rho_{w}$ is $1000 \mathrm{~kg} / \mathrm{m}^{3}$. The density of the glycerol-water mixture, based on $f$, is a linear interpolation between the densities of the pure substances.

$$
\rho=\rho_{w}(1-f)+\rho_{g} f
$$

The surface tension and contact angle are measured using a Krüss tensiometer and Cahn DCA-322 using the Wilhelmy slide method [12]. Experiments performed in this study all use mixtures containing high volume fractions of glycerol, thus the contact angle is assumed to be a constant and contact angle effects are not considered. The surface tension is found to vary linearly with the volumer fraction of glycerol, $f$, as,

$$
\sigma=72.4-8.3 f
$$

Viscosity measurements are performed using a Brookfield DVII+ viscometer equipped with a UL adapter which permits accurate viscosity measurements for viscosities as low as $10 \mathrm{cp}$. A constant temperature of $25^{\circ} \mathrm{C}$ is maintained by a Brookfield TC200 water bath. Measurements are performed at eight different volume fractions of glycerol ranging from 0.375 to 1.0 . Two polynomial curve fits, used over different domains, are used to express the viscosity as a function of glycerol volume fraction.

$$
\begin{gathered}
\mu=134.67 f^{3}-60.238 f^{2}+8.4509 f+1 \\
0.0<f<0.6875 \\
\mu=-53268 f^{5}+411091 f^{4}-934784 f^{3}+940459 f^{2}-442393 f+79755 \\
0.6875<f<1.0
\end{gathered}
$$

Equations (13)-(15) establish all the fluid properties in terms of $f$. These equations are substituted into Equation (12) to yield a single equation in terms of the volume fraction, $f$,

$$
0=\left(\frac{\left(\rho_{w}(1-f)+\rho_{g} f\right) B o}{g}\right)^{1 / 4}\left(\frac{C a}{\operatorname{Re}}\right)^{1 / 2}(72-8 f)^{3 / 4}-\mu(f)
$$

For a given set of non-dimensional parameters, Equation (16) can be solved nu- 
merically to determine $f$. Subsequently, Equations (13)-(15) are used to determine the fluid properties. These are then used in Equations (10)-(11) to determine $u$ and $L$. Finally, the volume flow rate, $Q$, is determined using the average velocity and the flow area at $R=50.8 \mathrm{~mm}(2.0 \mathrm{in}$.), as

$$
Q=u(2 \pi r L)
$$

The solution is performed over the range of non-dimensional parameters achievable by the experimental apparatus. The term $\mathrm{Ca} /$ Re in Equation (16) makes it convenient to create charts for the independent physical parameters vs. Bo with different curves for values of $\mathrm{Ca} / \mathrm{Re}$ shown in Figures 6-8. In Figure 6, it is observed that the fluid composition remains fairly constant for a given $\mathrm{Ca} / \mathrm{Re}$. In Figure 7, the gap width is seen to depend almost completely on $B o$. These charts are useful to quickly determine a set of physical experimental parameters based on desired non-dimensional parameters. To perform an experiment, the Reynolds, Bond, and Capillary numbers are first selected, then from Figure 6 the fluid composition is obtained. The gap-width is obtained from Figure 7, and the flow rate is set to the value prescribed in Figure 8.

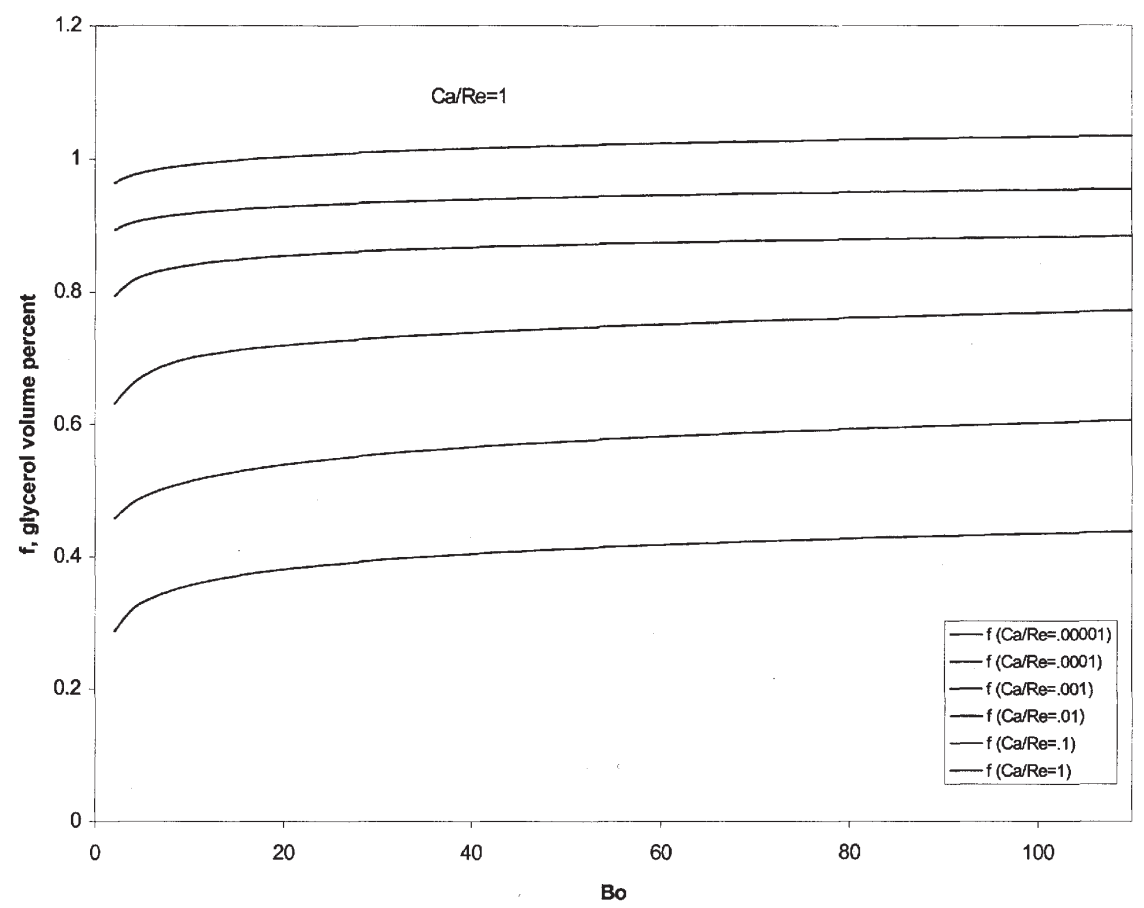

Figure 6. Volume fraction of glycerol vs. Bond number for different $\mathrm{Ca} / \mathrm{Re}$ ratios. 


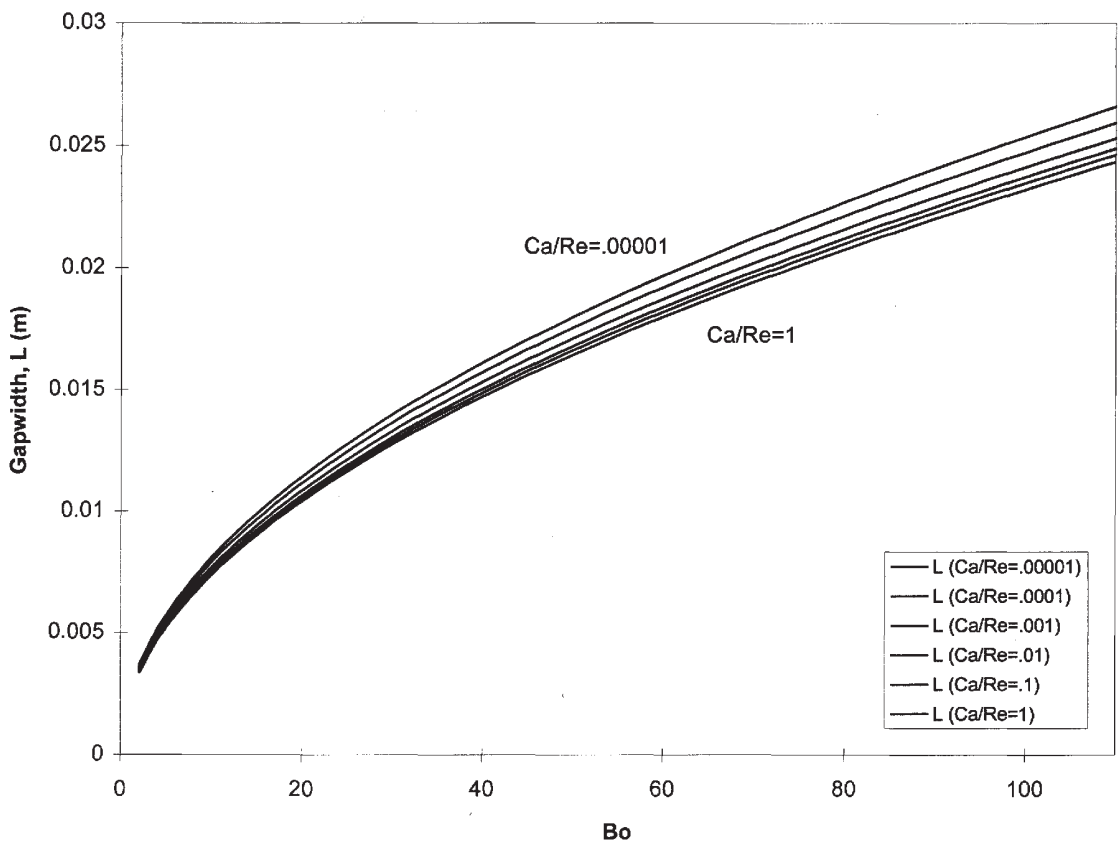

Figure 7. Gap-width vs. Bond number for different $\mathrm{Ca} /$ Re ratios.

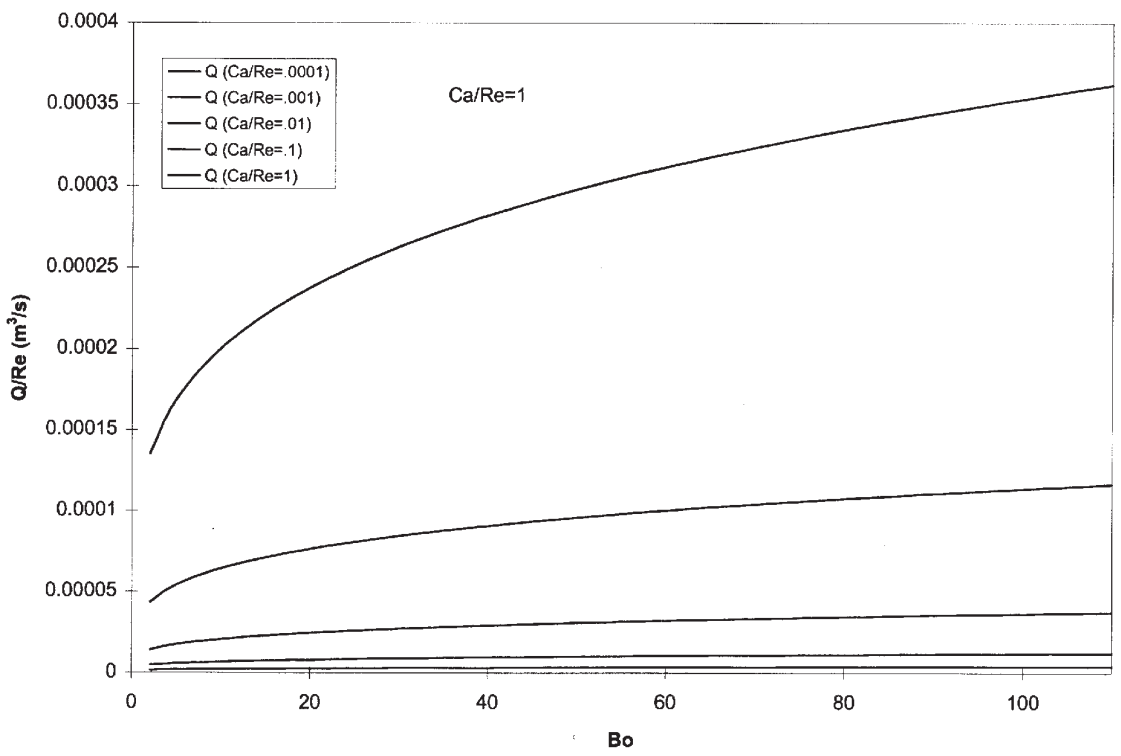

Figure 8. Volume flow rate/Re vs. Bond number of different $\mathrm{Ca} /$ Re ratios. 


\section{RESULTS}

Eleven sets of mold filling experiments are performed at various Bond and Reynolds numbers. In each set, spreading is measured at 50.8, 101.6, and 152.4 millimeters $(2,4$, and 6 in.) away from the inlet gate as described earlier. In all these eleven sets of experiments, the Capillary number is kept constant at 0.02 . Each filling experiment is repeated between 4 to 8 times, and the measured spreading values are given with a $95 \%$ confidence interval. Table 1 shows the Bond and Reynolds number of the eleven sets of mold filling experiments. Experiments are also performed at $B o=12.75$; however, at this Bond number, the bottom of the fluid front exits the mold cavity before any of the top sensors are contacted, thus resulting in "infinite" spreading. The experimental setup is capable of achieving higher Reynolds numbers at higher Bond number, but $\mathrm{Re}=4.0$ is the maximum at which repeatable data can be collected. The lower two Bond numbers have an upper Reynolds number limit of 2.0 due to flow rate limitations of the pump for the higher viscosities.

Spreading results for the eleven sets of experiments are depicted in Figure 9. There are three groups of three curves, representing the three radii, $R$, at each of the three Bond numbers. The largest errors are observed near $R=152.4 \mathrm{~mm}$ (6.0 in.) at the highest Bond number, where spreading is the highest. In all nine curves, spreading is seen to vary linearly over the range of Reynolds numbers studied. In most of these experiments, two orders of magnitude change in Reynolds number did not significantly affect spreading, as shown in Figure 9. However, Bond number is observed to significantly affect spreading at all Reynolds numbers. Figure 9 shows that spreading increases by an order of magni-

Table 1. Bond and Reynolds numbers used for eleven sets of mold filling experiments. Capillary number held constant at $\mathbf{0 . 0 2}$.

\begin{tabular}{ccc}
\hline Experiment & Re & Bo \\
\hline 1 & 0.04 & 4.35 \\
2 & 0.04 & 5.75 \\
3 & 0.04 & 8.20 \\
4 & 1.0 & 4.35 \\
5 & 1.0 & 5.75 \\
6 & 1.0 & 8.20 \\
7 & 2.0 & 4.35 \\
8 & 2.0 & 5.75 \\
9 & 2.0 & 8.20 \\
10 & 3.0 & 8.20 \\
11 & 4.0 & 8.20 \\
\hline
\end{tabular}




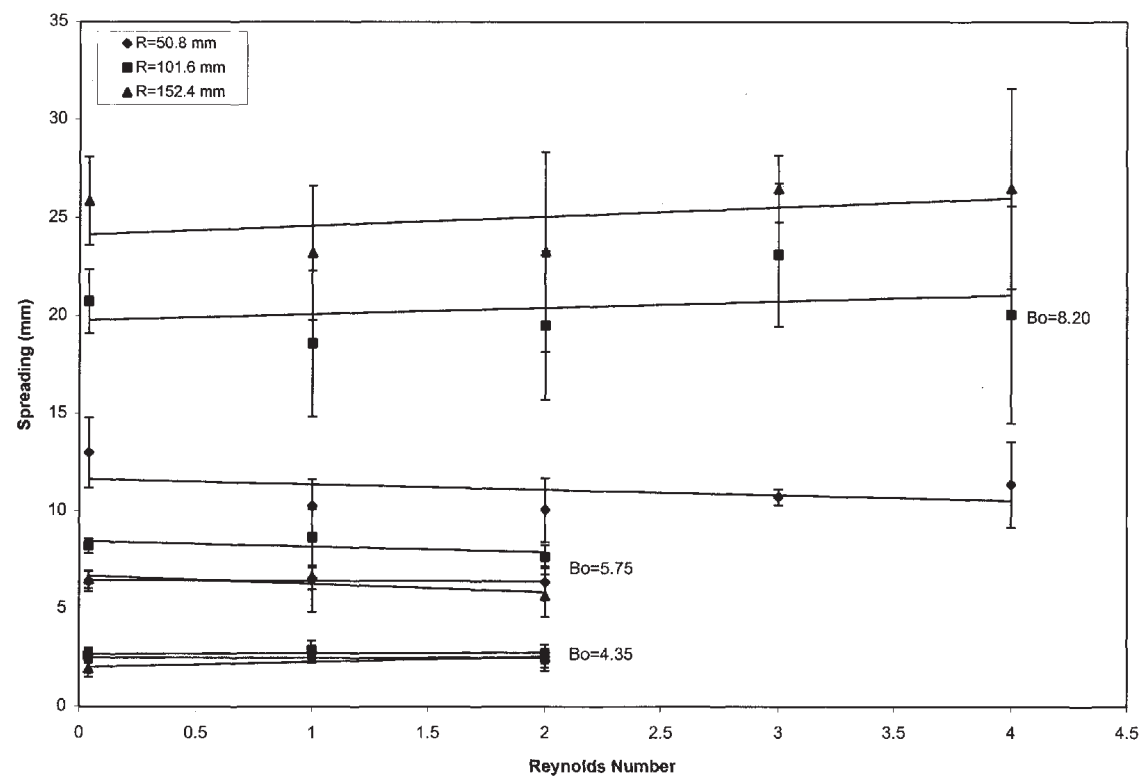

Figure 9. Spreading vs. Reynolds number at each radius for three Bond numbers (Bo $=4.35$, 5.75, and 8.20), $\mathrm{Ca}=0.02$.

tude due to a small increase in Bond number at all Reynolds numbers.

At $B o=8.2$, spreading is observed to be the least near the inlet, and increases as the fluid decelerates radially through the cavity. At $B o=5.75$ however, spreading is nearly identical on the $R=50.8 \mathrm{~mm}$ ( $2 \mathrm{in}$.) and $R=152.4 \mathrm{~mm}$ ( $6 \mathrm{in}$.) lines, and noticeably higher at $R=101.6 \mathrm{~mm}$ ( 4 in.). At $B o=4.35$ the same behavior is observed at $R=101.6 \mathrm{~mm}$ ( $4 \mathrm{in}$.). The increase in spreading at $R=101.6 \mathrm{~mm}$ ( $4 \mathrm{in}$.) at these Bond numbers is pronounced enough to be visually observed through the Plexiglas mold walls. This phenomenon is believed to be due to inlet effects as spreading is measured to be at the order of inlet diameter.

Figures 10-12 contain sets of spreading data obtained from different mold radii as a function of Bond number. In these figures, the error bars are the same as in Figure 9, thus they are not included for clarity. In each figure, the variation of spreading at different Bond numbers is shown for three different Reynolds numbers. In Figure 10, inlet effects at $R=50.8 \mathrm{~mm}$ ( 2 in.) are observed for the lowest Reynolds number, i.e., $\mathrm{Re}=0.04$. The spreading for $\mathrm{Re}=1.0$ and $\mathrm{Re}=2.0$ increases with Bond number and have a slightly negative radius of curvature. These curves demonstrate the previously observed trend that Reynolds number has minimal effect on spreading, in particular at low Bo numbers. The $\mathrm{Re}=0.04$ curve matches the others at the lower two Bond numbers as well, but is observed to deviate from the 


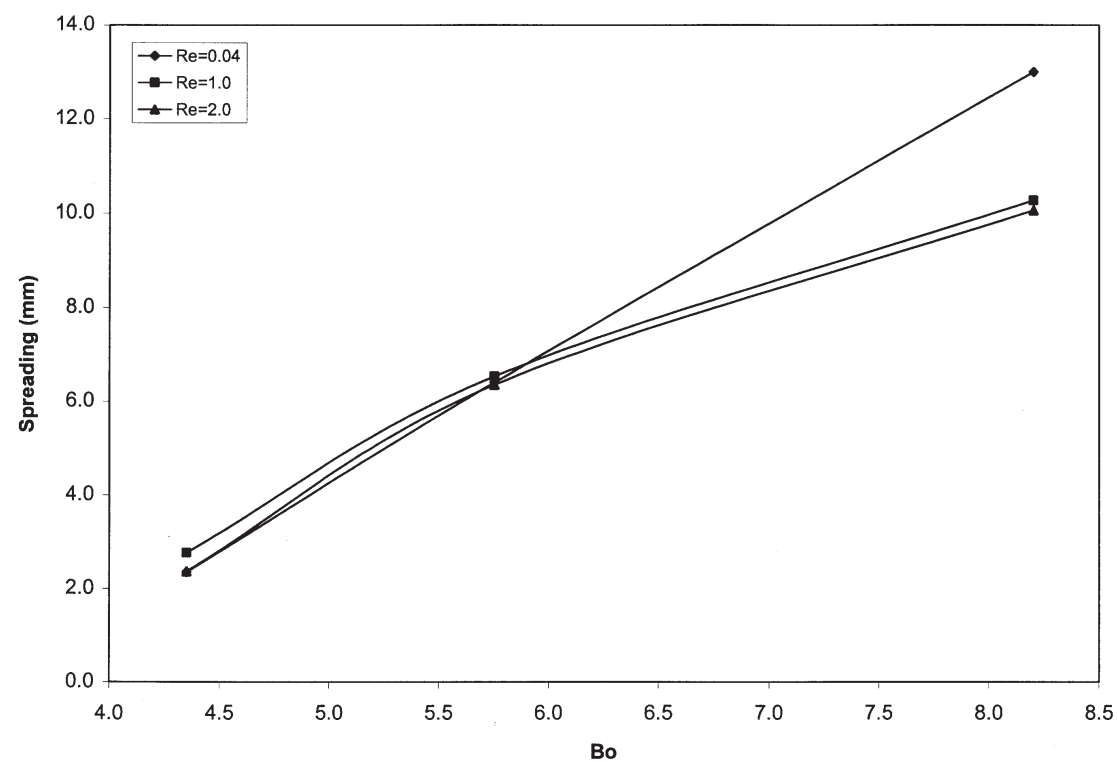

Figure 10. Spreading vs. Bond number for different Reynolds numbers at $\mathrm{R}=50.8 \mathrm{~mm}(2.0$ in.), $\mathrm{Ca}=0.02$.

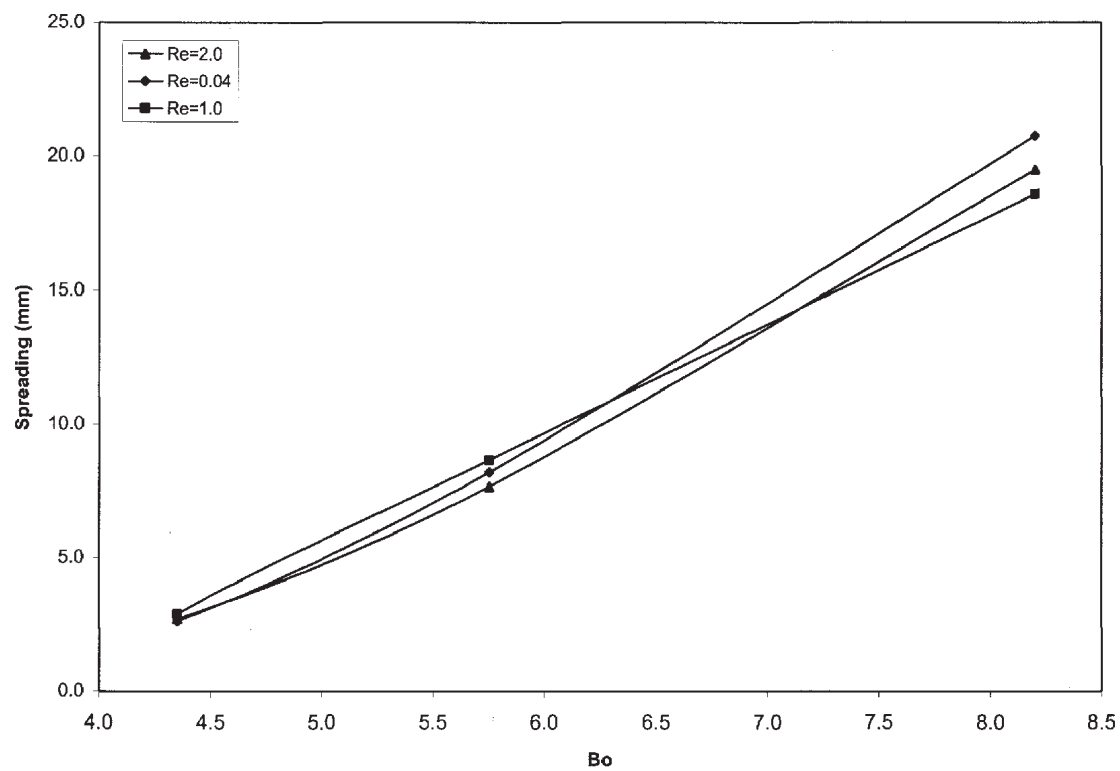

Figure 11. Spreading vs. Bond number for different Reynolds numbers at $\mathrm{R}=101.6 \mathrm{~mm}(4.0$ in.), $\mathrm{Ca}=0.02$. 


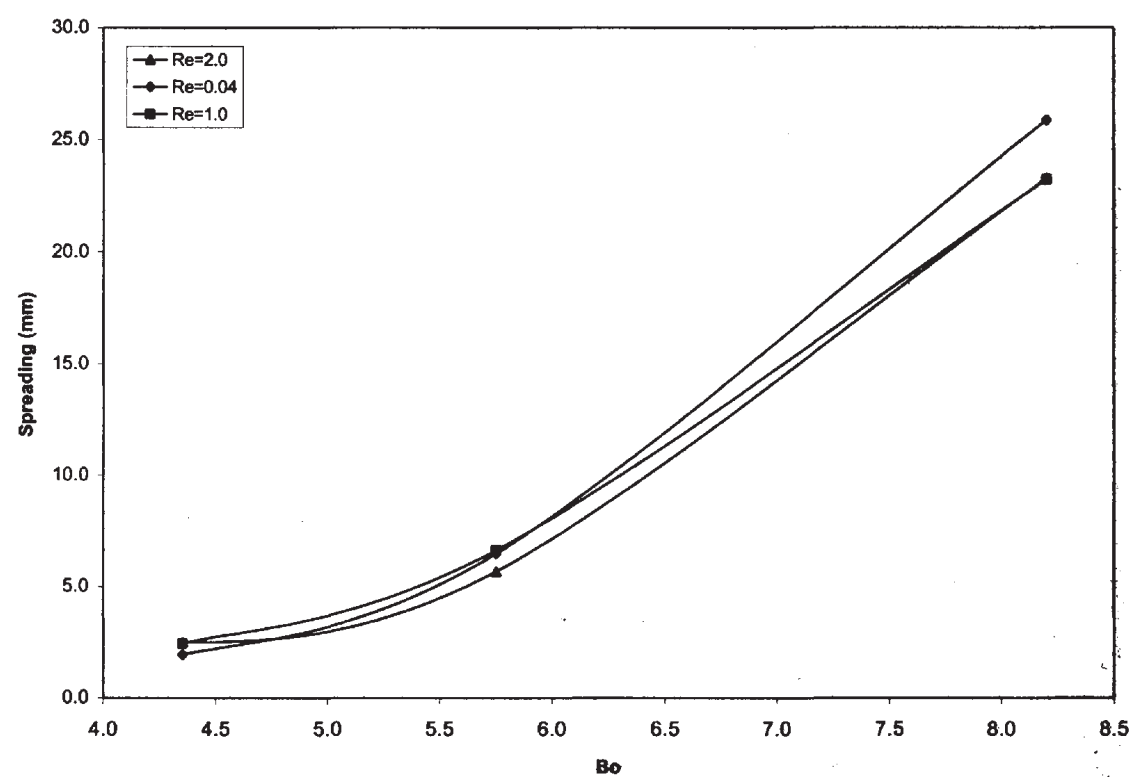

Figure 12. Spreading vs. Bond number for different Reynolds numbers at $\mathrm{R}=152.4 \mathrm{~mm}(6.0$ in.), $\mathrm{Ca}=0.02$.

others as the Bond number is increased. This is due to the inlet effect where a very slow flow at a high Bond number runs along the bottom mold wall before the fluid ever contacts the top mold wall. An interesting extension of this behavior is observed near this range of non-dimensional parameters, when the fluid spreads several inches along the bottom wall before the top touches, at which point the bottom of the fluid front slows down considerably for a brief instant while the top rapidly progresses ahead.

In Figure 11, spreading measurements are shown at $R=101.6 \mathrm{~mm}$ ( 4 in.), farther from the inlet. There is still a slight inlet effect visible for $B o=8.7$, but in general the curves have condensed and are similar for each Reynolds number. Spreading at this radius is observed to increase by almost an order of magnitude when the Bond number is doubled. The curves for these Reynolds numbers are nearly linear. The data from $R=152.4 \mathrm{~mm}$ (6 in.), depicted in Figure 12, exhibits a similar significant increase in spreading with Bond number, however in this case the curves are concave indicating around one-inch spreading at $R=152.4 \mathrm{~mm}$ (6 in.).

Similar mold filling experiments are performed holding the Bond number constant at 5.75, and the Reynolds number constant at 1.0, while varying the Capillary number between 0.006 and 0.2 . Results from these experiments are presented in Figure 13, with the spreading measurements drawn with a $95 \%$ confidence inter- 


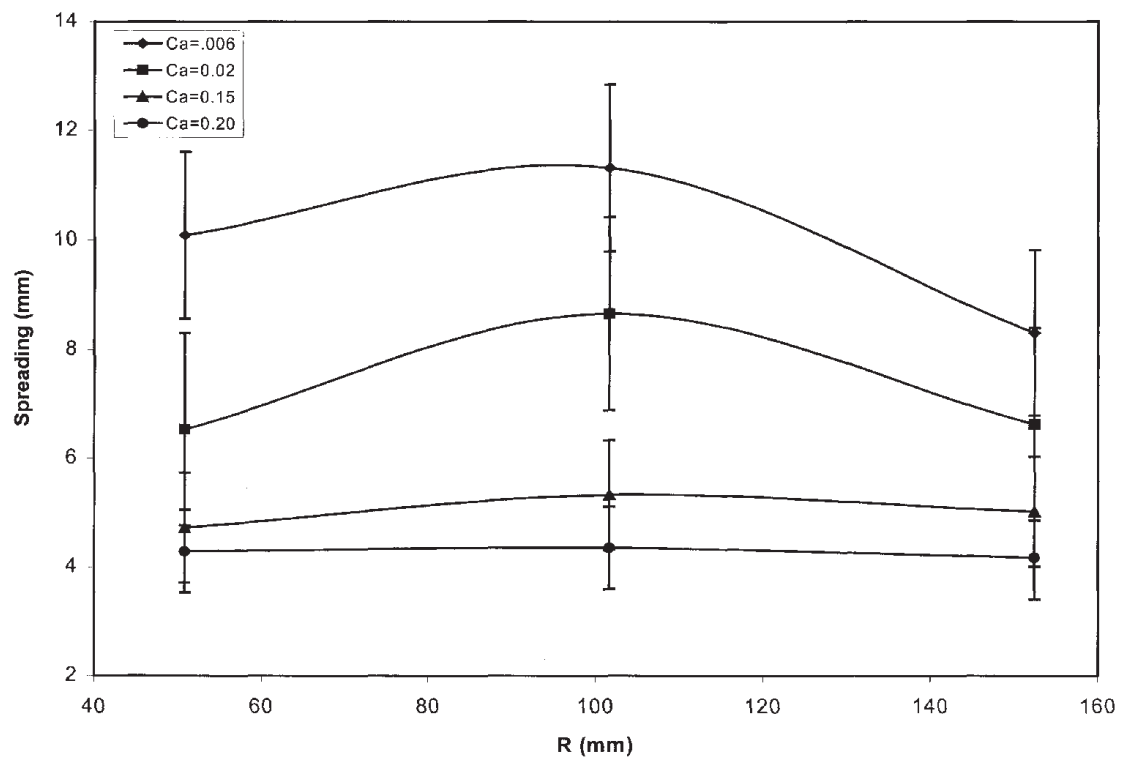

Figure 13. Spreading vs. radius, $\mathrm{R}$, for different Capillary numbers at $\mathrm{Re}=1.0$, Bo $=5.75$.

val. Figure 13 displays the general trend that spreading decreases with increasing Capillary number. Increasing the Capillary number translates to increasing both the volume flow rate and the fluid viscosity in terms of physical parameters. The dependence of spreading on Capillary number is less pronounced than the dependence on Bond number. The reduction in spreading is approximately 50\%, as Capillary number is increased nearly two orders of magnitude. The graphs of spreading vs. radius demonstrate the phenomena observed in the previous data set, where spreading increases from the inlet to a maximum at $R=101.6 \mathrm{~mm}$ (4 in.) at which point it begins to decrease again.

\section{CONCLUSIONS}

Spreading of the fluid front during the filling of a center-gated disk is characterized over a variety of fill conditions. A method is presented to determine the independent process parameters to isolate the effect of non-dimensional parameters governing the fluid motion. Spreading is found to remain nearly constant over the range of Reynolds numbers considered. In addition, spreading is observed to decrease with increasing Capillary number. Bond number is shown to be the primary parameter affecting the spreading dynamics in a mold filling process. Doubling the Bond number increases spreading amount by an order of magnitude throughout the mold cavity. Inlet effects on spreading results are also apparent at low 
Reynolds and high Bond numbers. In molding polymeric parts, the gravity effects may dominate the fluid front kinematics at high Bond numbers. These effects need to be considered in the flow analysis and numerical simulation of mold filling with low-viscosity polymers.

\section{ACKNOWLEDGEMENT}

This work has been supported by the NASA Microgravity Materials Research Program under Grant NAG8-1271.

\section{REFERENCES}

1. Behrens, R.A., Crochet, M.J., Denson, C.D. and Metzner, A.B., "Transient Free-Surface Flows: Motion of a Fluid Advancing in a Tube," AIChE Journal, 33(7), pp. 1178-1186, 1987.

2. Jansons, K.M., "Moving Contact Lines at Non-Zero Capillary Number," J. Fluid Mech., 167, pp. 393-407, 1986.

3. Dussan, E.B. and Davis, S.H., "On the Motion of a Fluid-Fluid Interface along a Solid Surface," $J$. Fluid Mech., 65(1), pp. 71-95, 1974.

4. Rame, E. and Garoff, S., "Micropscopic and Macroscopic Dynamic Interface Shapes and the Interpretation of Dynamic Contact Angles," J. Colloid and Interface Sci., 177, 234-244, 1996.

5. Gennes, P.G., Hua, X. and Levinson, P., "Dynamics of Wetting: Local Contact Angles," J. Fluid Mech., 212, pp. 55-63, 1989.

6. Ström, G., Fredriksson, M., Steinus, P. and Radoev, B., "Kinetics of Steady State Wetting," J. Colloid and Interface Sci., 134, 107-116, 1989.

7. Blake, J.W., "Studies in Reaction Injection Mold Filling," Ph.D. Thesis, University of Minnesota, 1987.

8. Blake, J.W. and Macosko, C.W. "The Kinematics of Fountain Flow in Mold-Filling," AIChE Journal, 33(7), pp. 1168-1177, 1987.

9. Chen, Q., Rame, E. and Garoff, S., "The Breakdown of Asymptotic Hydrodynamic Models of Liquid Spreading at Increasing Capillary Number," Phys. Fluids, 7(11), 2631-2639, 1995.

10. Hocking, L.M., "Rival Contact-Angle Models and the Spreading of Drops," J. Fluid Mech., 239, pp. 671-681, 1991.

11. Brosius, D. and Wadsworth, M., "Resin Transfer Molding of a Complex Composite Aircraft Structure," in Composites in Manufacturing, Ed. Strong, A.B., pp. 111-128, Society of Manufacturing Engineers, 1991.

12. Hwa, M.J., "Interfacial Properties of Modified Glass Fibers in Thermoset and Model Resins," Masters Thesis, University of Oklahoma, 1998. 